\title{
Model of nanocrystal formation in solution by burst nucleation and diffusional growth
}

\author{
Daniel T. Robb and Vladimir Privman
}

\section{Supporting Information Section S1: Determining the large-time \\ behavior of $\alpha, K, \rho$, and $L$ in the Gaussian Ansatz.}

The asymptotic large-time behavior of the width $\alpha$, the peak location $K$, and the amplitude parameter $\rho$ of the Gaussian Ansatz (see Fig. 5) can be determined by inserting the assumed Gaussian form,

$$
P_{G}(n, t)=\rho(t) c_{0} \exp \left[-(\alpha(t))^{2}(n-K(t))^{2}\right]
$$

into the asymptotic form of the growth equation for the supercritical distribution,

$$
\frac{\partial P_{n}}{\partial t}=\frac{z^{2}}{2}\left(\frac{1}{2} \frac{\partial^{2}}{\partial n^{2}}-\frac{\partial}{\partial n}\right) P_{n}
$$

In the resulting expression, equating the coefficients of $n^{2}$ yields $-2 \alpha^{-3}(d \alpha / d t)=z^{2}$, which has the solution $\alpha=\left(z^{2} t+\Upsilon\right)^{-1 / 2}$, with $\Upsilon$ a constant. (The time dependence of $\alpha, K$, and $\rho$ are suppressed for brevity). Equating coefficients of $n^{1}$ gives

$$
4 K \frac{d \alpha}{d t}+2 \alpha \frac{d K}{d t}=z^{2} \alpha-2 z^{2} K \alpha^{3}
$$


Substituting $\alpha=\left(z^{2} t+\Upsilon\right)^{-1 / 2}$ reduces Eq. (S1.3) to $d K / d t=z^{2} / 2$. Thus the asymptotic form is the straightforward linear relationship $K=\Phi+\frac{z^{2}}{2} t$, with $\Phi$ a constant . Lastly, equating the $n^{0}$ (constant) coefficients, and simplifying, yields

$$
\frac{d \rho}{d t}=\alpha \rho\left(2 \frac{d \alpha}{d t} K^{2}+2 \alpha K \frac{d K}{d t}+z^{2} \alpha^{3} K^{2}-\frac{z^{2}}{2} \alpha-z^{2} \alpha K\right)
$$

Using $2 d \alpha / d t=-z^{2}\left(z^{2} t+\Upsilon\right)^{-3 / 2}=-z^{2} \alpha^{3}$ and $d K / d t=z^{2} / 2$, Eq. (S1.4) simplifies to $d \rho / d t=-z^{2} \alpha^{2} \rho / 2$, which has solution $\rho=\Omega\left(z^{2} t+\Upsilon\right)^{-1 / 2}$.

The constants $\Upsilon, \Phi, \Omega$ cannot be determined by the asymptotic analysis. We summarize these large-time behaviors as

$$
\alpha \simeq 1 / \sqrt{z^{2} t}, \quad K \simeq z^{2} t / 2, \quad \rho \simeq \Omega / \sqrt{z^{2} t} .
$$

To determine the asymptotic behavior of the peak offset $L(t) \equiv N(t)-K(t)$, illustrated in Fig. 5, recall that total matter is conserved. Thus, as time increases, the amount of matter

$$
M(t) \equiv \int_{N(t)}^{\infty} n P_{G}(n, t) d n
$$

in the supercritical cluster distribution must approach a constant value, equal to the initial total matter less the matter that remains in the thermal distribution as $c \rightarrow c_{0}$. Substituting Eq. (S1.1) for $P_{G}(n, t)$ into Eq. (S1.6) gives 


$$
\begin{aligned}
M & =\int_{N}^{\infty} n c_{0} \rho \exp \left(-\alpha^{2}(n-K)^{2}\right) d n \\
& =\frac{c_{0} \rho}{2 \alpha^{2}} \int_{(\alpha L)^{2}}^{\infty} \exp (-u) d u+\frac{c_{0} \rho K}{\alpha} \int_{\alpha L}^{\infty} \exp \left(-v^{2}\right) d v,
\end{aligned}
$$

where the integral was rearranged by use of the change of variables $v=\alpha(n-K)$, and the definition $L=N-K$. (The time-dependences of $M$ and $L$ are suppressed for brevity.) It is straightforward to show that if the quantity $\alpha L$ does not diverge (as $t \rightarrow \infty$ ), then $M$ diverges; therefore, $\alpha L$ must diverge. To determine the nature of the divergence, we can replace the second integral in Eq. (S1.7) with the first term in the expansion, $\int_{\alpha L}^{\infty} \exp \left(-u^{2}\right) d u \approx \exp \left(-\alpha^{2} L^{2}\right)\left((2 \alpha L)^{-1}-\left(4 \alpha^{3} L^{3}\right)^{-1}+\ldots\right)$. This results in the asymptotic expression

$$
M=\frac{c_{0} \rho}{2 \alpha^{2}} \exp \left(-\alpha^{2} L^{2}\right)\left(1+\frac{K}{L}\right)
$$

Using the asymptotic forms, Eq. (S1.5), one can verify that $M$ approaches a constant if the peak offset at large times behaves as

$$
L \simeq \Psi \sqrt{t \ln t}\left[1-O\left(\frac{\ln \ln t}{\ln t}\right)\right]
$$

The constant $\Psi$ cannot be determined by the asymptotic analysis. 